\title{
Efficacy of Oral Nicorandil to Prevent Contrast-Induced Nephropathy in Patients with Chronic Renal Dysfunction Undergoing an Elective Coronary Procedure
}

\author{
Zeyuan Fan Yang Li Hanhua Ji Xinwen Jian \\ Department of Cardiovascular Diseases, Civil Aviation General Hospital, Civil Aviation \\ Clinical Medical College of Peking University, Beijing, China
}

\section{Keywords}

Nicorandil · Contrast-induced nephropathy $\cdot$ Chronic renal dysfunction

\begin{abstract}
Objectives: This prospective, randomized study was to investigate the role of nicorandil in the prevention of contrast-induced nephropathy $(\mathrm{CIN})$ in patients with chronic renal dysfunction undergoing an elective coronary procedure. Methods: A total of 252 eligible patients were enrolled in this study and allocated into the control group $(n=125)$ or nicorandil group $(n=$ 127). Both groups received the standard hydration treatment, and patients in the nicorandil group were orally administrated $10 \mathrm{mg}$ of nicorandil (t.i.d.) beginning 2 days before and continuing for 2 days after an elective coronary procedure. Serum creatinine (SCr) and cystatin C (CysC) were measured at $24 \mathrm{~h}$ before and 24, 48, and $72 \mathrm{~h}$ after the procedure. The occurrences of $\mathrm{CIN}$ and adverse events within 1 year were recorded. Results: The nicorandil group had relatively lower SCr and CysC levels and a higher eGFR at 24 and $48 \mathrm{~h}$ after the procedure than the control group $(p<0.05)$. The incidence of CIN was significantly decreased in the nicorandil group compared to the control group. The multivariate logistic regression model revealed that nicorandil treatment was an independent protective factor for CIN (OR 0.669, $95 \% \mathrm{Cl} 0.522-0.857, p=0.001)$. The multivariate COX proportional hazard model showed that nicorandil treatment was an independent protective predictor for adverse events (HR 0.881, 95\% Cl 0.781-0.993, $p=0.037)$. Conclusions: Nicorandil could exhibit a protective effect against $\mathrm{CIN}$ in patients with chronic renal dysfunction undergoing an elective coronary procedure and reduce the adverse events within 1 year after the procedure, which is superior to hydration treatment only.




\section{Kidney \\ Blood Pressure \\ Research}

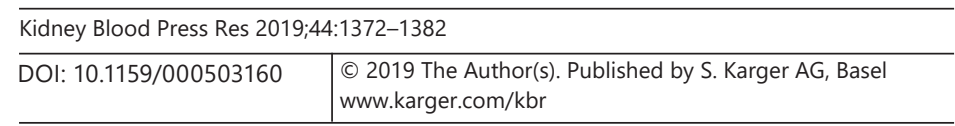

Fan et al.: Nephroprotective Effect of Nicorandil against CIN

\section{Introduction}

Contrast-induced nephropathy (CIN), a form of acute kidney injury, is a serious complication that occurs after exposure to the iodinated contrast media [1-3]. With the everincreasing use of iodinated contrast media with coronary interventional procedures, the incidence of CIN is increasing, especially in patients with poor renal function [4-6]. Recent studies have recognized that CIN is associated with long-term adverse events and mortality $[7,8]$. Several approaches, such as hydration, $\mathrm{N}$-acetylcysteine, sodium bicarbonate, and statins, have been studied to prevent CIN, but their therapeutic effects still need further investigation [9].

Nicorandil (2-nicotinamidoethyl-nitrate ester) is a hybrid compound derived from an adenosine triphosphate-sensitive potassium channel (K-ATP channel) opener and a nitric oxide donor, and has been widely used in the treatment of angina pectoris and acute heart failure $[10,11]$. Nicorandil exhibits a vasodilatory effect on the coronary vasculature and a pharmacologic preconditioning effect to protect the heart from ischemia $[10,12]$. Moreover, nicorandil could ameliorate ischemia-reperfusion injury in the rat kidney due to its ischemic preconditioning and anti-oxidant properties $[13,14]$. Several studies have investigated the potential role of nicorandil in CIN prevention, but the results remain inconsistent. To the best of our knowledge, there has been no previous study with a long-term follow-up to evaluate the nephroprotective effect of nicorandil against CIN. This randomized, prospective trial was to assess the short-term and long-term protective effect of nicorandil against CIN and associated adverse events in patients with chronic renal dysfunction undergoing an elective coronary procedure.

\section{Materials and Methods}

\section{Study Population}

A total of 278 consecutive patients with renal dysfunction scheduled for coronary angiography or percutaneous coronary intervention (PCI) at our institution from January 2016 to January 2018 were considered for participation in our study. Renal dysfunction was defined as an estimated glomerular filtration rate (eGFR) $\leq 60 \mathrm{~mL} / \mathrm{min} / 1.73 \mathrm{~m}^{2}$ calculated using the Chronic Kidney Disease Epidemiology Collaboration (CKD-EPI) formula. Exclusion criteria were the following: end-stage renal disease; a history of kidney transplantation; left ventricular ejection fraction (LVEF) $<35 \%$ or New York Heart Association (NYHA) IV class; acute myocardial infarction; previous contrast media exposure within 1 week; allergy to contrast medium or nicorandil, and the administration of other medications, such as $\mathrm{N}$-acetylcysteine, metformin, and sodium bicarbonate to prevent CIN. End-stage renal disease was defined as eGFR $<15 \mathrm{~mL} / \mathrm{min} / 1.73 \mathrm{~m}^{2}$ or the need for long-term dialysis or kidney transplantation. LVEF was measured by echocardiography and calculated from the end-diastolic and end-systolic volumes calculated using the Simpson method from two orthogonal apical views.

\section{Study Protocol}

The study was a prospective, open-labeled, randomized controlled trial. Eligible patients were randomly allocated into either control or nicorandil groups, using a balanced block randomization method. Patients in the nicorandil group received $10 \mathrm{mg}$ of nicorandil three times per day (t.i.d.), from 2 days before to 2 days after an elective coronary procedure. An elective coronary procedure was performed according to the standard technique by a radial or femoral approach. All enrolled patients received standard hydration with an intravenous 


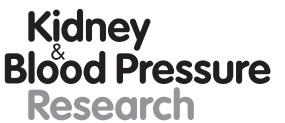

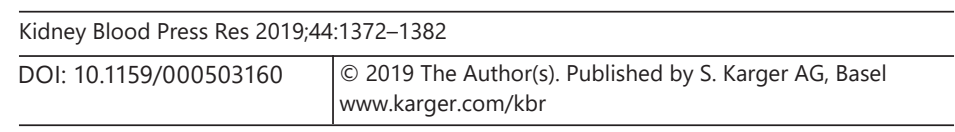

Fan et al.: Nephroprotective Effect of Nicorandil against CIN

infusion of $0.9 \%$ saline at a rate of $1 \mathrm{~mL} / \mathrm{kg} / \mathrm{h}$ for $6 \mathrm{~h}$ before and $12 \mathrm{~h}$ after an elective coronary procedure. The nonionic contrast agent (Iopamidol injection, BRACCO Sine Ltd., China) was used for all patients.

Blood samples were collected at $24 \mathrm{~h}$ before and 24,48 , and $72 \mathrm{~h}$ after the procedure. Serum creatinine (SCr) was measured by an enzymatic method (Creatinine Diagnosis Kit, Roche Diagnostics, Germany), and Cystatin C (CysC) was determined by the immune turbidimetric method (Cystatin C Determination Kit, Mike Biotechnology Co. Ltd, China). CIN was defined as an SCr increase of $\geq 25 \%$ and/or $\geq 0.5 \mathrm{mg} /$ day within $72 \mathrm{~h}$ after exposure to the contrast medium.

\section{Follow-Up}

The median follow-up period of the study was 12.8 months. The follow-up endpoints were adverse events, including all-cause mortality, stroke, non-fatal myocardial infarction, percutaneous coronary revascularization, coronary artery bypass graft surgery, congestive heart failure, pulmonary edema, and end-stage renal disease. If more than one adverse event occurred in the same patient, the first event was used for the analysis. The follow-up data were obtained from electronic medical records and/or telephone interview with the patients or patients' caregivers. During the follow-up period, all the patients continued their regular medications without interruption.

\section{Statistical Analysis}

The incidence of CIN in the control group was approximate at $20 \%$, while the incidence of CIN in the nicorandil group was hypothesized to be $5 \%$ according to the previous studies $[15,16]$. The sample size was determined based on a two-sample, two-sided inequality test. The required number of participants was 93 per group, with the power of the test set at $90 \%$ and the significance level at 0.05 . Taking into account the patients lost to follow-up (estimated at $20 \%$ ), the number of patients enrolled in this study was at least 112 per group.

The continuous data are expressed as the mean \pm standard deviation (SD) or median (interquartile range). The Kolmogorov-Smirnov test was used to analyze the normal distribution of continuous data. The Student $t$ test, Mann-Whitney U test, or Wilcoxon rank-sum test was used for the comparisons of continuous variables. The categorical data were compared using $\chi^{2}$ tests. Univariate and multivariate logistic regression analyses were used to identify the factors for CIN, while univariate and multivariate Cox proportional hazard analyses were carried out to determine the independent predictors for adverse events during 1 year of follow-up. Covariates with a $p$ value $<0.10$ after univariate analysis were entered into multivariate regression models. The cumulative adverse event rates were estimated by the Kaplan-Meier analysis and statistical differences were carried out using the log-rank test. The statistical significance was considered as a 2-tailed $p<0.05$. Statistical analyses were performed using SPSS version 22.0.

\section{Results}

Of the 278 patients enrolled in this study, 8 (2.88\%) were excluded because of incomplete medical data, and another $5(1.80 \%)$ were withdrawn from the study at their own request. A total of 265 patients (95.32\%) were randomized to either receive nicorandil and hydration (nicorandil group, $n=133$ ) or hydration only (control group, $n=132$ ). During the 1-year follow-up period, 6 patients from the nicorandil group and 7 patients from the control group missed the follow-up. Consequently, 127 patients in the nicorandil group and 125 patients in the control group completed the study. The study flowchart is depicted in Figure 1. 


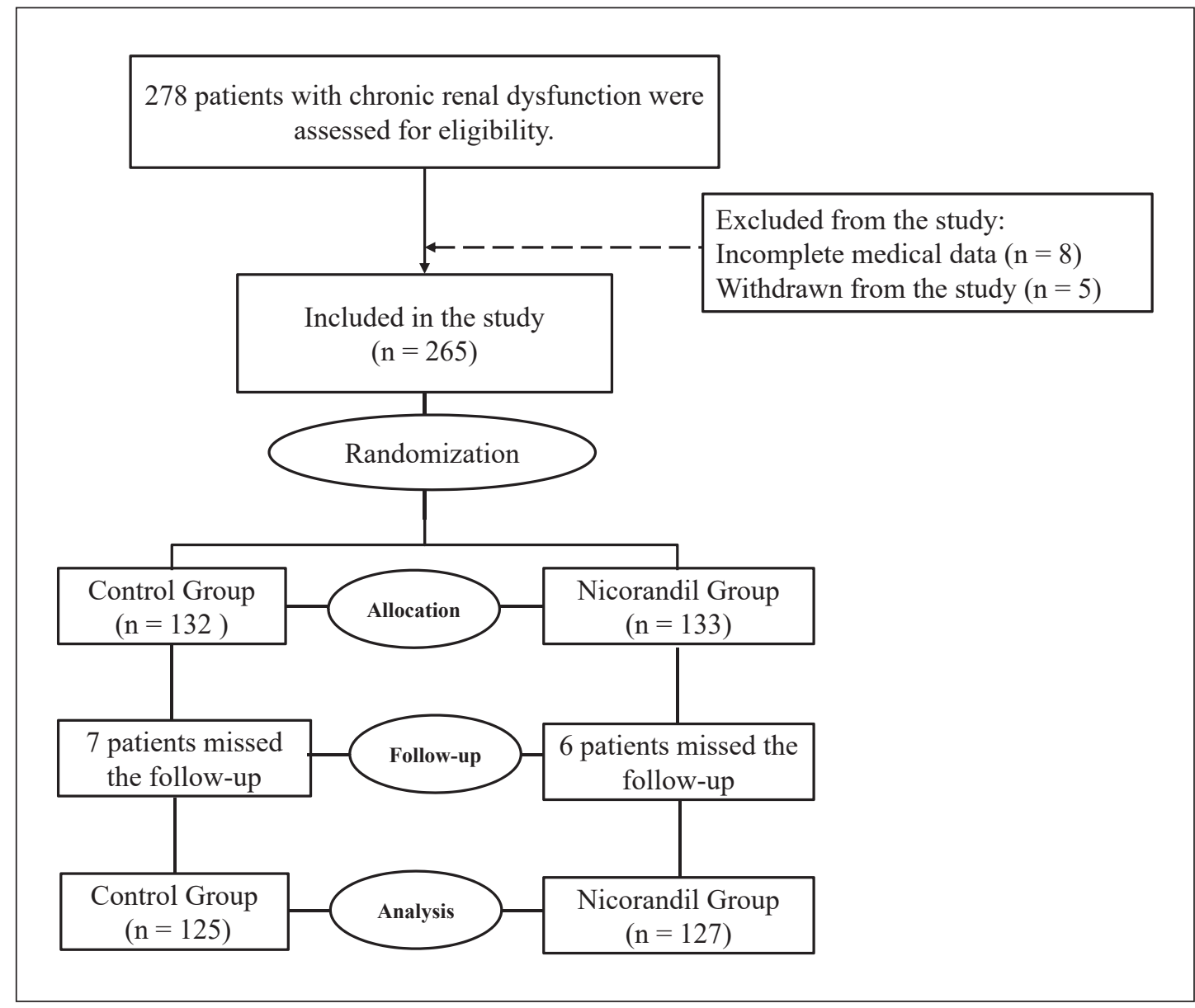

Fig. 1. Study flowchart.

\section{Patients' Baseline Characteristics}

The baseline clinical characteristics of the patients are summarized in Table 1 and the angiographic and procedural characteristics are presented in Table 2. No significant differences were observed between the two groups in age, gender, underlying diseases, laboratory results, medications, and angiographic/procedural features.

\section{Changes in Indicators of Renal Function}

As the enrolled patients had poor renal functions, both groups had relatively high basal $\mathrm{SCr}$ and $\mathrm{CysC}$, and low eGFR. Additionally, there was no significant difference in the volume of contrast medium used between the nicorandil group $(128.3 \pm 37.2 \mathrm{~mL})$ and the control group $(122.8 \pm 35.9 \mathrm{~mL} ; p=0.234)$.

Baseline and post-procedural levels of SCr, eGFR, and CysC are compared between the two groups in Table 3. The levels of SCr and CysC of both groups were increased at 24 and $48 \mathrm{~h}$ after an elective coronary procedure, whereas no significant differences were found at $72 \mathrm{~h}$ after procedure, compared to the baseline levels. The levels of SCr and CysC peaked at 48 and $24 \mathrm{~h}$, respectively. eGFR levels of both groups declined at 24 and $48 \mathrm{~h}$, and then increased to baseline levels at $72 \mathrm{~h}$. The levels of SCr and CysC at 24 and $48 \mathrm{~h}$ were lower, whereas the levels of eGFR at 24 and $48 \mathrm{~h}$ were higher in the nicorandil group compared with those of the control group. 
Table 1. Patients' baseline clinical characteristics

\begin{tabular}{lccc}
\hline & $\begin{array}{l}\text { Nicorandil group } \\
(n=127)\end{array}$ & $\begin{array}{l}\text { Control group } \\
(n=125)\end{array}$ & $p$ value \\
\hline Age, years & $62.25 \pm 16.63$ & $65.87 \pm 17.62$ & 0.095 \\
Male & $76(59.84)$ & $67(53.60)$ & 0.317 \\
BMI & $24.35 \pm 5.87$ & $23.78 \pm 5.98$ & 0.446 \\
Smoking & $31(24.41)$ & $27(21.6)$ & 0.596 \\
Hypertension & $68(53.54)$ & $62(49.6)$ & 0.531 \\
Diabetes & $81(63.78)$ & $75(60)$ & 0.537 \\
Laboratory results & & & \\
$\quad$ Hemoglobin, g/L & $127.52 \pm 21.52$ & $131.56 \pm 23.37$ & 0.155 \\
$\quad$ Total cholesterol, mmol/L & $4.46 \pm 1.21$ & $4.37 \pm 1.19$ & 0.552 \\
$\quad$ LDL-C, mmol/L & $2.74 \pm 0.79$ & $2.71 \pm 0.81$ & 0.766 \\
$\quad$ hsCRP, mg/dL & $7.16 \pm 3.42$ & $7.41 \pm 3.25$ & 0.553 \\
$\quad$ BNP, $\mu$ g/L & $72.71 \pm 17.28$ & $75.63 \pm 18.33$ & 0.194 \\
LVEF, \% & $51.39 \pm 10.35$ & $53.58 \pm 12.77$ & 0.136 \\
Medications & & & \\
$\quad$ Aspirin & $108(85.04)$ & $101(80.80)$ & 0.371 \\
Clopidogrel & $83(65.35)$ & $79(63.20)$ & 0.721 \\
ACEI/ARB & $46(36.22)$ & $44(35.20)$ & 0.866 \\
Calcium-channel blocker & $50(39.37)$ & $56(44.80)$ & 0.383 \\
StBlockers & $61(48.03)$ & $53(42.40)$ & 0.369 \\
\hline
\end{tabular}

Data are presented as the mean \pm SD or $n(\%)$. BMI, body mass index; LDL-C, low-density lipoprotein cholesterol; hsCRP, high-sensitivity C-reactive protein; BNP, brain natriuretic peptide; LVEF, left ventricular ejection fraction.

Table 2. Procedural variables

\begin{tabular}{lccc}
\hline & $\begin{array}{l}\text { Nicorandil group } \\
(n=127)\end{array}$ & $\begin{array}{c}\text { Control group } \\
(n=125)\end{array}$ & $p$ value \\
\hline Hospital days & $6.85 \pm 1.33$ & $7.18 \pm 1.65$ & 0.081 \\
PCI & $74(58.27)$ & $82(65.60)$ & 0.231 \\
Target lesion & $72(56.69)$ & $65(52.00)$ & 0.713 \\
$\quad$ LAD & $32(25.20)$ & $33(26.40)$ & \\
$\quad$ LCX & $23(18.11)$ & $27(21.60)$ & \\
RCA & $1.63 \pm 0.91$ & $1.72 \pm 0.86$ & 0.421 \\
Surgents per patient, $n$ & $51.37 \pm 10.38$ & $53.41 \pm 11.25$ & 0.136 \\
Contrast medium volume, $\mathrm{mL}$ & $128.39 \pm 37.25$ & $122.81 \pm 35.92$ & 0.227 \\
\hline
\end{tabular}

Data are presented as the mean \pm SD or $n(\%)$. PCI, percutaneous coronary intervention; LAD, left anterior descending artery; LCX, left circumflex artery; RCA, right coronary artery.

Incidence of CIN

Overall, CIN was diagnosed in 27 (10.71\%) of the 252 patients within $72 \mathrm{~h}$ after an elective coronary procedure, which consisted of 8 patients in the nicorandil group and 19 patients in the control group. The incidence of CIN was lower in the nicorandil group than the control group (6.30 vs. $15.20 \%, p=0.022$; Fig. 2 ). 
Table 3. Comparisons of markers of renal function between the two groups

\begin{tabular}{lccc}
\hline & $\begin{array}{l}\text { Nicorandil group } \\
(n=127)\end{array}$ & $\begin{array}{l}\text { Control group } \\
(n=125)\end{array}$ & $p$ value \\
\hline SCr, umol/L & & & \\
Baseline & $121.22 \pm 22.35$ & $118.75 \pm 26.18$ & 0.421 \\
$24 \mathrm{~h}$ & $124.35 \pm 26.45$ & $131.36 \pm 27.89$ & 0.042 \\
$48 \mathrm{~h}$ & $129.87 \pm 25.78$ & $137.19 \pm 30.18$ & 0.039 \\
$72 \mathrm{~h}$ & $118.74 \pm 23.69$ & $123.16 \pm 27.37$ & 0.171 \\
eGFR, $\mathrm{mL} / \mathrm{min} / 1.73 \mathrm{~m}^{2}$ & $59.32 \pm 19.31$ & $61.75 \pm 22.56$ & 0.359 \\
Baseline & $56.73 \pm 15.64$ & $52.66 \pm 14.82$ & 0.035 \\
$24 \mathrm{~h}$ & $49.35 \pm 13.98$ & $45.7 \pm 12.61$ & 0.031 \\
$48 \mathrm{~h}$ & $58.29 \pm 17.64$ & $57.31 \pm 18.77$ & 0.670 \\
$72 \mathrm{~h}$ & $1.17 \pm 0.28$ & $1.12 \pm 0.29$ & 0.165 \\
CysC, mg/L & $1.38 \pm 0.44$ & $1.52 \pm 0.58$ & 0.032 \\
Baseline & $1.36 \pm 0.51$ & $1.49 \pm 0.38$ & 0.023 \\
$24 \mathrm{~h}$ & $1.19 \pm 0.34$ & $1.2 \pm 0.37$ & 0.823 \\
$48 \mathrm{~h}$ & & & \\
\hline $72 \mathrm{~h}$ & & & \\
\hline
\end{tabular}

Data are presented as the mean \pm SD. SCr, serum creatinine; eGFR, estimated glomerular filtration rate; CysC, cystatin C.

Fig. 2. Incidence of CIN. The nicorandil group had a higher CIN incidence than the control group.

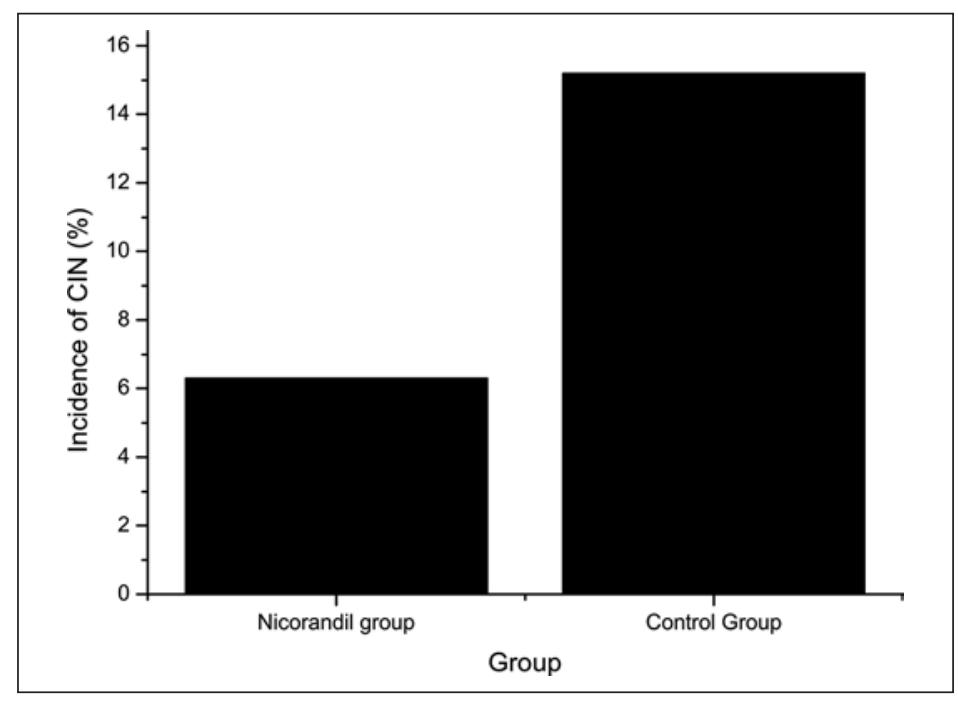

Independent Predictors for the Development of CIN

Univariate analysis results revealed that age, diabetes, contrast volume, and nicorandil treatment were associated with CIN. In the multivariate logistic regression analysis (Table 4), nicorandil treatment was found to be an independent protective factor for CIN (OR 0.669, $95 \%$ CI $0.522-0.857, p=0.001$ ), while diabetes was also an independent risk factor for CIN (OR 1.429, 95\% CI 1.065-1.918, $p=0.017$ ).

\section{Adverse Events during the 1-Year Follow-Up}

During the follow-up period, of the 252 patients, 60 (24 in the nicorandil group and 36 in the control group) experienced adverse events. The adverse events of the two groups are summarized in Table 5. Overall, the nicorandil group had fewer adverse events (18.1 vs. 
Table 4. Multivariate logistic regression analysis for the independent predictors of CIN

\begin{tabular}{llllll}
\hline Variable & $\beta$ & Wald & OR & $95 \%$ CI & $p$ value \\
\hline Age & 0.362 & 2.368 & 1.436 & $0.906-2.277$ & 0.124 \\
Diabetes & 0.357 & 5.647 & 1.429 & $1.065-1.918$ & 0.017 \\
Nicorandil & -0.402 & 10.163 & 0.669 & $0.522-0.857$ & 0.001 \\
Contrast volume & 0.571 & 1.589 & 1.770 & $0.728-4.301$ & 0.207 \\
\hline
\end{tabular}

Table 5. Distribution of adverse events in the two groups

\begin{tabular}{llr}
\hline & $\begin{array}{l}\text { Nicorandil group } \\
(n=127)\end{array}$ & $\begin{array}{l}\text { Control group } \\
(n=125)\end{array}$ \\
\hline Nonfatal MI & $3(2.36)$ & $5(4.00)$ \\
Revascularization & $6(4.72)$ & $10(8.00)$ \\
Ischemic stroke & $4(3.15)$ & $6(4.80)$ \\
Coronary artery bypass graft surgery & $1(0.79)$ & $2(1.60)$ \\
Congestive heart failure & $5(3.94)$ & $5(4.00)$ \\
Pulmonary edema & $2(1.57)$ & $2(1.60)$ \\
End-stage kidney disease & $2(1.57)$ & $4(3.20)$ \\
Cardiac death & $1(0.79)$ & $2(1.60)$ \\
\hline
\end{tabular}

Fig. 3. Kaplan-Meier curve of the cumulative incidence of adverse events during the follow-up period.

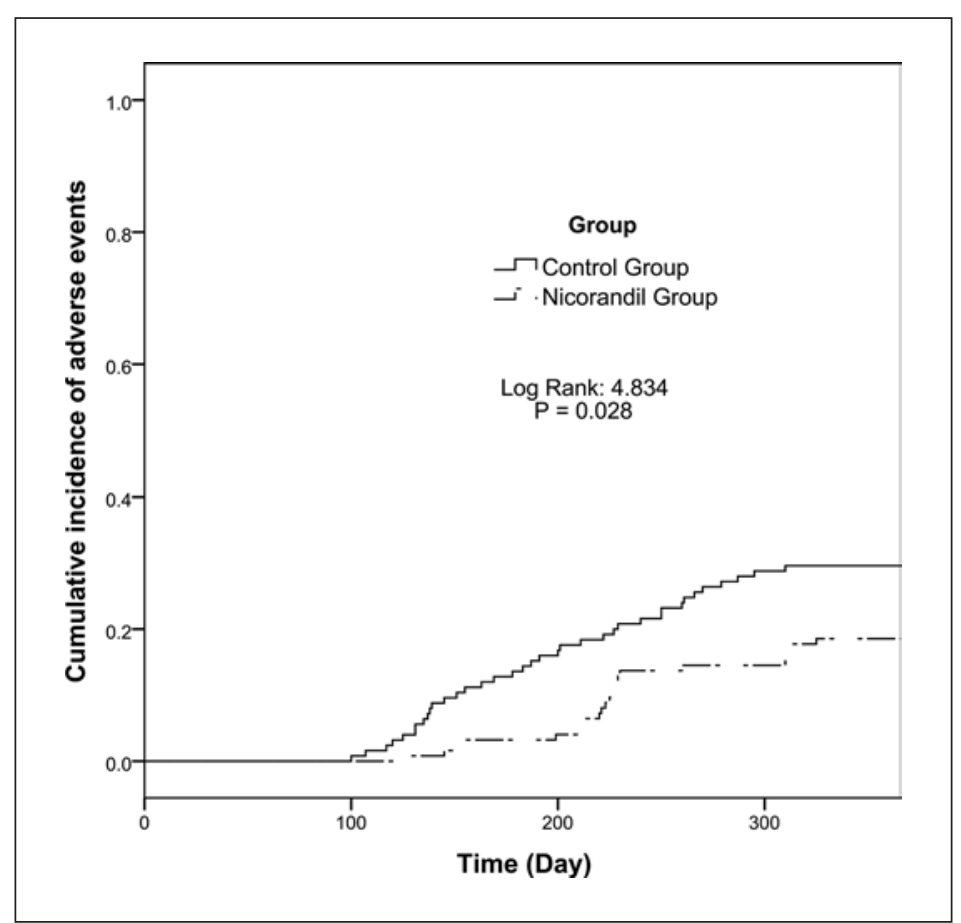

$28.8 \%, p=0.032$ ) than the control group. Kaplan-Meier curve analysis revealed that the cumulative adverse event rate was significantly lower in the nicorandil group compared to the control group (log-rank: 4.834, $p=0.028$; Fig. 3). Meanwhile, Spearman's correlation analysis revealed that the CIN occurrence was positively correlated with adverse events $(r=$ $0.415, p<0.001)$. 
Table 6. Multivariate Cox proportional hazards analysis for adverse events

\begin{tabular}{lrllll}
\hline & $\beta$ & Wald & HR & $95 \% \mathrm{CI}$ & $p$ value \\
\hline Age & 0.162 & 5.098 & 1.176 & $1.022-1.353$ & 0.024 \\
Hypertension & 0.372 & 2.524 & 1.451 & $0.917-2.295$ & 0.112 \\
Diabetes & 0.118 & 4.118 & 1.125 & $1.004-1.261$ & 0.042 \\
Nicorandil & -0.127 & 4.329 & 0.881 & $0.781-0.993$ & 0.037 \\
\hline
\end{tabular}

\section{Independent Predictors for Adverse Events}

In the univariate Cox proportional hazard analysis, age, hypertension, diabetes, and nicorandil treatment were found to be significantly associated with adverse events. After adjusting for covariates, age (HR 1.176, 95\% CI 1.022-1.353, $p=0.024$ ), diabetes (HR 1.125, 95\% CI 1.004-1.261, $p=0.042$ ), and nicorandil treatment (HR 0.881, 95\% CI 0.781-0.993, $p=0.037)$ remained significant predictors for adverse events in the multivariate Cox regression (Table 6).

\section{Discussion}

The present study evaluated nicorandil treatment in patients with chronic renal dysfunction undergoing an elective coronary procedure to determine whether the treatment could prevent CIN. The major findings of our study were that nicorandil could reduce the incidence of CIN and also decrease the occurrence of adverse events over 1 year of follow-up.

The exact pathophysiology of CIN remains unclear. Several mechanisms may contribute to the pathogenesis of CIN, including the direct cytotoxic effects of contrast media on renal epithelial cells because of its high osmolality, activated tubuloglomerular feedback, impaired production of nitric oxide, adenosine, endothelin, prostaglandin, and angiotensin resulting in imbalance between renal vasodilation and vasoconstriction, and increased renal interstitial pressure, which were all attributed to the hypoxia of the medulla and acute tubular necrosis [17-20]. Regarding possible mechanisms, many preventive measures have been investigated for protection against CIN. Hydration, $\mathrm{N}$-acetylcysteine, sodium bicarbonate, statins, and acetylcysteine were all studied, but many of them still remain controversial [21-23].

Nicorandil, a nicotinamide derivative, is widely used in the treatment of angina and heart failure. Nicorandil exerted cardioprotective properties by preventing reperfusion injury via promoting ischemic preconditioning, protecting against myocardial ischemia through its vasodilatory effects as a nitric oxide donor, and improving microvascular circulation [10-12]. Large-scale population-based studies, such as the Impact of Nicorandil in Angina (IONA) study [24] and Japanese Coronary Artery Disease (JCAD) study [25], confirmed that nicorandil could significantly reduce the incidence of major adverse cardiovascular events in patients with coronary heart diseases. Additionally, several basic studies demonstrated the nephroprotective potential of nicorandil. Zhang et al. [14] reported that pretreatment with nicorandil could protect against renal ischaemia-reperfusion injury by inhibiting the production of inflammatory cytokines and restoring the expression of KIR6.2 in a rat model. Also, Tamura et al. [26] found that nicorandil could reduce albuminuria and alleviate chronic renal injury through blocking oxidative stress.

Several studies have investigated the potential role of nicorandil in CIN prevention, but the results are inconsistent. Nawa et al. [27] reported that nicorandil continuously infused 


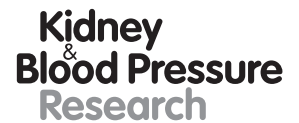

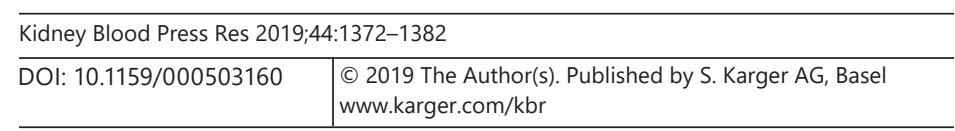

Fan et al.: Nephroprotective Effect of Nicorandil against CIN

intravenously at $1 \mathrm{~mL} / \mathrm{kg} / \mathrm{h}$ beginning at $4 \mathrm{~h}$ before the procedure and continuing for $24 \mathrm{~h}$ after it could have a favorable nephroprotective effect against CIN in patients with poor renal function. Fan et al. [16] found that orally administrated nicorandil (10 mg, t.i.d.) could prevent CIN in patients with renal insufficiency. Zhang et al. [28] reported that prophylactic administration of nicorandil could prevent CIN in patients with moderate renal insufficiency undergoing PCI. Meta-analyses conducted by both Li et al. [29] and Wang et al. [30] confirmed that nicorandil can reduce the incidence of CIN in patients exposed to contrast medium. However, another recent study undertaken by Ko et al. [31] reported that prophylactic intravenous infusion of $12 \mathrm{mg}$ of nicorandil prior to the coronary angiography could not decrease the incidence of CIN in patients with renal dysfunction undergoing coronary angiography. The exact reason for the discrepancy between these studies was unclear, which might be due to the differences in the administration schedule of nicorandil. The mean elimination half-life of nicorandil is very short, reported to be $0.8-1.3 \mathrm{~h}$ in both normal volunteers and patients with impaired renal function [32], while the elimination half-life of contrast medium is relatively long (approx. $2 \mathrm{~h}$ in healthy individuals and $4 \mathrm{~h}$ in patients with mild renal disease) [33]. Therefore, the administration of nicorandil after the procedure was very critical to prevent against CIN. In our study, the patients received oral nicorandil (10 mg t.i.d.) beginning 2 days before and continuing for 2 days after the procedure. Our results showed that continuous nicorandil treatment could significantly reduce the incidence of CIN, which is in accordance with the studies by Nawa et al. [27] and Fan et al. [16].

Recently, increasing attention has been paid to the relationship between CIN and longterm clinical outcomes in patients undergoing an elective coronary procedure. CIN may lead to end-stage renal disease and major adverse cardiovascular events [34]. Abe et al. [35] found that CIN was associated with increased mortality in patients who underwent PCI with chronic kidney diseases. Solomon et al. [3] demonstrated that CIN was associated with increased long-term adverse events, including revascularization, coronary artery bypass graft surgery, myocardial infarction, and mortality, in renally impaired patients undergoing coronary angiography. In our study, CIN showed a significant positive correlation with adverse events in 1 year, which was consistent with previous studies. Additionally, the incidence of adverse events was decreased in the nicorandil group and nicorandil was found to be a protective predictor for the presence of adverse events, which was probably associated with the cardioprotective and renoprotective properties of nicorandil. Moreover, to the best of our knowledge, our study is the first to report 1-year follow-up outcomes of prophylactic administration of nicorandil in patients with chronic renal dysfunction undergoing an elective coronary procedure.

There were several limitations to this study. This was not a blinded study. A larger, multicenter and double-blinded study would be needed to confirm the effectiveness of nicorandil in preventing CIN in patients with chronic renal dysfunction. Most of the subjects recruited into this study had mild-stage renal dysfunction, meaning we cannot conclude the nephroprotective effect of nicorandil against CIN in patients with severe renal dysfunction.

In conclusion, this study suggests that nicorandil has a nephroprotective effect against CIN in patients with chronic renal dysfunction undergoing an elective coronary procedure. Additionally, nicorandil could be helpful to decrease the incidence of adverse events after the procedure.

\section{Statement of Ethics}

Subjects have given their written informed consent. The study protocol was approved by the Ethics Committee of the Civil Aviation General Hospital. 


\section{Kidney \\ Blood Pressure \\ Research}

\begin{tabular}{l|l}
\hline Kidney Blood Press Res 2019;44:1372-1382 \\
\hline DOI: 10.1159/000503160 & $\begin{array}{l}\text { @ 2019 The Author(s). Published by S. Karger AG, Basel } \\
\text { www.karger.com/kbr }\end{array}$ \\
\hline
\end{tabular}

Fan et al.: Nephroprotective Effect of Nicorandil against CIN

\section{Disclosure Statement}

The authors declare that they have no competing interests.

\section{Author Contributions}

Z.F. was principally responsible for the conception and design of the study, and prepared the first draft of the manuscript. Y.L., H.J., and X.J. contributed to the data acquisition and performed the statistical analyses. Y.L. revised the manuscript. All authors read and approved the final manuscript prior to submission.

\section{References}

1 Dangas G, Iakovou I, Nikolsky E, Aymong ED, Mintz GS, Kipshidze NN, et al. Contrast-induced nephropathy after percutaneous coronary interventions in relation to chronic kidney disease and hemodynamic variables. Am J Cardiol. 2005 Jan;95(1):13-9.

2 Stacul F, van der Molen AJ, Reimer P, Webb JA, Thomsen HS, Morcos SK, et al.; Contrast Media Safety Committee of European Society of Urogenital Radiology (ESUR). Contrast induced nephropathy: updated ESUR Contrast Media Safety Committee guidelines. Eur Radiol. 2011 Dec;21(12):2527-41.

3 Solomon R, Dauerman HL. Contrast-induced acute kidney injury. Circulation. 2010 Dec;122(23):2451-5.

4 Rihal CS, Textor SC, Grill DE, Berger PB, Ting HH, Best PJ, et al. Incidence and prognostic importance of acute renal failure after percutaneous coronary intervention. Circulation. 2002 May;105(19):2259-64.

5 Jörres A, John S, Lewington A, ter Wee PM, Vanholder R, Van Biesen W, et al.; ad-hoc working group of ERBP. A European Renal Best Practice (ERBP) position statement on the Kidney Disease Improving Global Outcomes (KDIGO) Clinical Practice Guidelines on Acute Kidney Injury: part 2: renal replacement therapy. Nephrol Dial Transplant. 2013 Dec;28(12):2940-5.

6 Kim MJ, Choi HS, Oh SH, Lee HC, Kim CS, Choi JS, et al. Impact of acute kidney injury on clinical outcomes after ST elevation acute myocardial infarction. Yonsei Med J. 2011 Jul;52(4):603-9.

7 Solomon RJ, Mehran R, Natarajan MK, Doucet S, Katholi RE, Staniloae CS, et al. Contrast-induced nephropathy and long-term adverse events: cause and effect? Clin J Am Soc Nephrol. 2009 Jul;4(7):1162-9.

8 Kim JH, Yang JH, Choi SH, Song YB, Hahn JY, Choi JH, et al. Predictors of outcomes of contrast-induced acute kidney injury after percutaneous coronary intervention in patients with chronic kidney disease. Am J Cardiol. 2014 Dec;114(12):1830-5.

9 Weisbord SD, Palevsky PM. Strategies for the prevention of contrast-induced acute kidney injury. Curr Opin Nephrol Hypertens. 2010 Nov;19(6):539-49.

10 Taira N. Nicorandil as a hybrid between nitrates and potassium channel activators. Am J Cardiol. 1989 Jun; 63(21):18J-24J.

11 Ponikowski P, Voors AA, Anker SD, Bueno H, Cleland JG, Coats AJ, et al. 2016 ESC guidelines for the diagnosis and treatment of acute and chronic heart failure. Rev Esp Cardiol. 2016 Dec;69(12):1167.

12 Duerden MG. Role of nicorandil in ischaemic preconditioning. Lancet. 2002 Dec;360(9348):1887.

13 Shimizu S, Saito M, Kinoshita Y, Ohmasa F, Dimitriadis F, Shomori K, et al. Nicorandil ameliorates ischaemiareperfusion injury in the rat kidney. Br J Pharmacol. 2011 May;163(2):272-82.

14 Zhang YJ, Zhang AQ, Zhao XX, Tian ZL, Yao L. Nicorandil protects against ischaemia-reperfusion injury in newborn rat kidney. Pharmacology. 2013;92(5-6):245-56.

15 Li WH, Li DY, Qian WH, Liu JL, Xu TD, Zhu H, et al. Prevention of contrast-induced nephropathy with prostaglandin E1 in high-risk patients undergoing percutaneous coronary intervention. Int Urol Nephrol. 2014 Apr; 46(4):781-6.

16 Fan Y, Wei Q, Cai J, Shi Y, Zhang Y, Yao L, et al. Preventive effect of oral nicorandil on contrast-induced nephropathy in patients with renal insufficiency undergoing elective cardiac catheterization. Heart Vessels. 2016 Nov;31(11):1776-82.

17 Jorgensen AL. Contrast-induced nephropathy: pathophysiology and preventive strategies. Crit Care Nurse. 2013 Feb;33(1):37-46.

18 Geenen RW, Kingma HJ, van der Molen AJ. Contrast-induced nephropathy: pharmacology, pathophysiology and prevention. Insights Imaging. 2013 Dec;4(6):811-20.

19 Lenhard DC, Frisk AL, Lengsfeld P, Pietsch H, Jost G. The effect of iodinated contrast agent properties on renal kinetics and oxygenation. Invest Radiol. 2013 Apr;48(4):175-82.

20 Davenport MS, Khalatbari S, Cohan RH, Dillman JR, Myles JD, Ellis JH. Contrast material-induced nephrotoxicity and intravenous low-osmolality iodinated contrast material: risk stratification by using estimated glomerular filtration rate. Radiology. 2013 Sep;268(3):719-28. 
21 Azzalini L, Spagnoli V, Ly HQ. Contrast-induced nephropathy: from pathophysiology to preventive strategies. Can J Cardiol. 2016 Feb;32(2):247-55.

22 Xu R, Tao A, Bai Y, Deng Y, Chen G. Effectiveness of N-Acetylcysteine for the Prevention of Contrast-Induced Nephropathy: A Systematic Review and Meta-Analysis of Randomized Controlled Trials. J Am Heart Assoc. 2016 Sep;5(9):5.

23 Agarwal SK, Mohareb S, Patel A, Yacoub R, DiNicolantonio JJ, Konstantinidis I, et al. Systematic oral hydration with water is similar to parenteral hydration for prevention of contrast-induced nephropathy: an updated meta-analysis of randomised clinical data. Open Heart. 2015 Oct;2(1):e000317.

24 Group IS; IONA Study Group. Effect of nicorandil on coronary events in patients with stable angina: the Impact Of Nicorandil in Angina (IONA) randomised trial. Lancet. 2002 Apr;359(9314):1269-75.

25 Horinaka S, Yabe A, Yagi H, Ishimitsu T, Yamazaki T, Suzuki S, et al.; JCAD Study Investigators. Effects of nicorandil on cardiovascular events in patients with coronary artery disease in the Japanese Coronary Artery Disease (JCAD) study. Circ J. 2010 Mar;74(3):503-9.

26 Tamura Y, Tanabe K, Kitagawa W, Uchida S, Schreiner GF, Johnson RJ, et al. Nicorandil, a K(atp) channel opener, alleviates chronic renal injury by targeting podocytes and macrophages. Am J Physiol Renal Physiol. 2012 Aug; 303(3):F339-49.

27 Nawa T, Nishigaki K, Kinomura Y, Tanaka T, Yamada Y, Kawasaki M, et al. Continuous intravenous infusion of nicorandil for 4 hours before and 24 hours after percutaneous coronary intervention protects against contrastinduced nephropathy in patients with poor renal function. Int J Cardiol. 2015 Sep;195:228-34.

28 Zhang P, Li WY, Yang SC, Fu NK, Liu XG, Zhang X, et al. Preventive effects of nicorandil against contrast-induced nephropathy in patients with moderate renal insufficiency undergoing percutaneous coronary intervention. Angiology. 2019 Apr. doi: 10.1177/0003319719841733.

29 Li S, Wang L, Liu Y, Hu Q. Preventive effect of nicorandil on contrast-induced nephropathy: a meta-analysis of randomised controlled trials. Intern Med J. 2018 Aug;48(8):957-63.

30 Wang X, Geng J, Zhu H, Xing C. Renoprotective effect of nicorandil in patients undergoing percutaneous coronary intervention: a meta-analysis of 4 randomized controlled trials. Oncotarget. 2018 Jan;9(14):1183745.

31 Ko YG, Lee BK, Kang WC, Moon JY, Cho YH, Choi SH, et al.; PRINCIPLE Investigators. Preventive effect of pretreatment with intravenous nicorandil on contrast-induced nephropathy in patients with renal dysfunction undergoing coronary angiography (PRINCIPLE Study). Yonsei Med J. 2013 Jul;54(4):957-64.

32 Molinaro M, Villa G, Regazzi MB, Salvadeo A, Segagni S, Rondanelli R, et al. Pharmacokinetics of nicorandil in patients with normal and impaired renal function. Eur J Clin Pharmacol. 1992;42(2):203-7.

33 Corradi A, Menta R, Cambi V, Maccarini P, Cerutti R. Pharmacokinetics of iopamidol in adults with renal failure. Arzneimittelforschung. 1990 Jul; 40(7):830-2.

34 Rear R, Bell RM, Hausenloy DJ. Contrast-induced nephropathy following angiography and cardiac interventions. Heart. 2016 Apr;102(8):638-48.

35 Abe M, Morimoto T, Akao M, Furukawa Y, Nakagawa Y, Shizuta S, et al. Relation of contrast-induced nephropathy to long-term mortality after percutaneous coronary intervention. Am J Cardiol. 2014 Aug;114(3):362-8. 